\title{
Matrix-isolation FTIR study of azidoacetone and azidoacetonitrile
}

\author{
Marcin Frankowski ${ }^{1}$, Manuel Algarra ${ }^{2}$, Paula Rodrigues ${ }^{3}$, Maria T. Barros ${ }^{3}$, \\ M. Natália D.S. Cordeiro ${ }^{4}$, Brigitte S. Fox ${ }^{1}$, Alice M. Smith-Gicklhorn ${ }^{1}$, \\ Martin K. Beyer ${ }^{1}$, Maria L. Costa ${ }^{2}$, and Vladimir E. Bondybey ${ }^{1}$ \\ ${ }^{1}$ Institute of Physical and Theoretical Chemistry, Technical University of Munich \\ Garching 85747, Germany \\ E-mail: bondybey@ch.tum.de; \\ frankowski@ch.tum.de \\ ${ }^{2}$ CEFITEC, Department of Physics, Fac. Sciences and Technology, Universidade Nova de Lisboa \\ Caparica P-2829-516, Portugal \\ ${ }^{3}$ CQFB, Department of Chemistry, Fac. Sciences and Technology, Universidade Nova de Lisboa, \\ Caparica P-2829-516, Portugal \\ ${ }^{4}$ REQUIMTE, Department of Chemistry, Fac. Sciences, Universidade do Porto \\ Porto 4169-007, Portugal
}

Azidoacetonitrile $\left(\mathrm{N}_{3} \mathrm{CH}_{2} \mathrm{CN}\right)$ and azidoacetone $\left(\mathrm{N}_{3} \mathrm{CH}_{2} \mathrm{COCH}_{3}\right)$ were studied by matrix-isolation FTIR spectroscopy in solid neon, argon and nitrogen. The IR spectra calculated using the density fuctional theoretical method are discussed in comparison with the experimental data. Significant broadening of the recorded azide bands indicate an awkward fit of these compounds into the solid environment. The strongest absorption is observed for both compounds in the regions of asymmetric and symmetric stretches of the $\mathrm{N}_{3}$ azide group. Strong band splittings in the $\mathrm{N}_{3}$ asymmetric stretch region can be most likely explained by very strong Fermi resonances with the $\mathrm{CN}$ stretch and combinations and overtones of the numerous lower frequency vibrational modes.

PACS: 67.40.Yv, 07.60.Vg

\section{Introduction}

Organic azides are useful reagents in many fields [1-3]. Their strongly exothermic reactions make them useful as propellants [4]. Their ability to form highly reactive nitrene intermediates that are capable of inserting into otherwise stable chemical bonds makes them important synthetic reagents, with for instance a number of potential uses in semiconductor technology [5].

The compounds were studied both in solution and in solid phase, and the relative volatility of some of them, for example azidoacetonitrile $\left(\mathrm{N}_{3} \mathrm{CH}_{2} \mathrm{CN}\right.$, in this work abbreviated as AzN) and azidoacetone $\left(\mathrm{N}_{3} \mathrm{CH}_{2} \mathrm{COCH}_{3}, \mathrm{AzC}\right)$, allows their studies in the gas phase [6]. The thermal decomposition of these two compounds was recently studied using ultraviolet pho- toelectron spectroscopy (UVPES) and matrix-isolation spectroscopy in solid nitrogen, as part of a general project on decomposition of aliphatic azides $[7,8]$. Theoretical calculations have been also carried out for conformers of $\mathrm{AzC}$ [9].

One can often obtain more insight and additional information about the interaction of isolated compounds with the solid medium by examining and comparing the spectra in several matrices. In the present paper we report the infrared spectra in solid neon, argon and nitrogen. We complement the experimental investigation by density functional theoretical (DFT) computations of their properties and structures, and discuss their spectra with the help of computed vibrational frequencies and intensities. 


\section{Experimental}

\section{Sample preparation}

Azidoacetonitrile was synthesized from chloroacetonitrile $\mathrm{ClCH}_{2} \mathrm{CN}$ and sodium azide $\left(\mathrm{NaN}_{3}\right)$ as given in [10]. Azidoacetone was prepared from chloroacetone $\mathrm{ClCH}_{2} \mathrm{COCH}_{3}$ and $\mathrm{NaN}_{3}$, according to the procedure described in [7].

The pre-mixed organic azides vapors with matrix $\mathrm{Ne}$, Ar and $\mathrm{N}_{2}$ gases were prepared at room temperature with an estimated concentration of 1:700 for all experiments. Neon samples were continuously deposited onto the copper substrate (coated by silver and $\mathrm{MgF}_{2}$ ) cooled to $6 \mathrm{~K}$ by a closed cycle helium cryostat, Leybold RGD 580. Argon and nitrogen samples were deposited by means of a pulsed valve at $12 \mathrm{~K}$ onto the $\mathrm{KCl}$ substrate mounted on the cryotip of an APD Cryogenics refrigerator. To improve the optical quality of the $\mathrm{N}_{2}$ matrix, deposition was started from $60 \mathrm{~K}$ and then the substrate was gradually cooled down to $12 \mathrm{~K}$ in $10 \mathrm{~min}$. In all experiments the deposition rate was kept in the range of $2.5-3 \mathrm{mmol} / \mathrm{h}$ for a deposition time of 2 hours.

\section{Spectroscopy}

The infrared spectra of both compounds, $\mathrm{N}_{3} \mathrm{CH}_{2} \mathrm{CN}$ and $\mathrm{N}_{3} \mathrm{CH}_{2} \mathrm{COCH}_{3}$, in solid $\mathrm{Ne}, \mathrm{Ar}$, and $\mathrm{N}_{2}$ were recorded with resolution of $0.06 \mathrm{~cm}^{-1}$ with a Bruker IFS 120 HR Fourier-transform spectrometer equipped with a glowbar light source, liquid nitrogen cooled HgCdTe (MCT) detector, and a $\mathrm{KBr}$ beam splitter. Typically at least 500 scans were averaged. The spectra were recorded immediately after deposition and then again after annealing of the samples, and after irradiation of the matrices with a tungsten lamp, a multi-line $\mathrm{UV} \mathrm{Ar}^{+}$ion laser and the fourth harmonic of a Nd:YAG laser.

\section{Results and discussion}

\section{Calculations}

The optimized structures and vibrational frequencies of azidoacetonitrile and azidoacetone were calculated using the B3LYP/6-31+G(d,p) hybrid DFT technique as implemented in the «Gaussian 98» suite of programs [11]. To check for errors due to the incomplete basis set, calculations using the larger B3LYP/6-311(3df,3pd) basis set were performed, which yielded no substantial changes from our previous results. Tables 1 and 2 summarize the results for the two most stable conformational minima, whose geometry is characterized by different values of the $\phi_{\text {NNCC }}$ dihedral angle. In the case of $\mathrm{AzC}$ the two local minima at $64^{\circ}$ (gauche) and $180^{\circ}$ (anti) are nearly isoenergetic, in $\mathrm{AzN}$ the $67^{\circ}$ gauche conformer is found to be considerably lower in energy than the $180^{\circ}$ anti isomer. The tables list the computed, unscaled vibrational frequencies of both molecules. The geometries of $\mathrm{AzN}$ and $\mathrm{AzC}$ conformers are illustrated in Fig. 1.

Table 1

Calculated frequencies of vibrational modes of azidoacetonitrile conformers (method: B3LYP / 6-31+G(d,p)

\begin{tabular}{|c|c|c|c|c|}
\hline \multicolumn{2}{|c|}{$\begin{array}{c}\text { Anti: dihedral angle } \\
\phi_{\mathrm{NNCC}}=180^{\circ}\end{array}$} & \multicolumn{2}{|c|}{$\begin{array}{l}\text { Gauche: dihedral } \\
\text { angle } \phi_{\mathrm{NNCC}}=67^{\circ}\end{array}$} & \multirow{2}{*}{$\begin{array}{l}\text { Approximate } \\
\text { assignment }\end{array}$} \\
\hline $\begin{array}{l}\text { frequency, } \\
\qquad \mathrm{cm}^{-1}\end{array}$ & $\begin{array}{l}\text { intensity, } \\
\mathrm{km} / \mathrm{mol}\end{array}$ & $\begin{array}{l}\text { frequency, } \\
\mathrm{cm}^{-1}\end{array}$ & $\begin{array}{l}\text { intensity, } \\
\mathrm{km} / \mathrm{mol}\end{array}$ & \\
\hline 34.9 & 1.3 & $\overline{55.7}$ & 5.5 & \\
\hline 145.2 & 9.2 & 193.1 & 7.7 & \\
\hline 260.2 & 1.6 & 258.6 & 0.6 & \\
\hline 358.6 & 0.6 & 351 & 0.1 & $\mathrm{C}-\mathrm{C} \equiv \mathrm{N}$ bend \\
\hline 519.4 & 2.7 & 523 & 0.1 & $\mathrm{C}-\mathrm{C} \equiv \mathrm{N}$ bend \\
\hline 544.9 & 6.9 & 556.3 & 8.7 & NNN bend \\
\hline 628.1 & 5 & 687.3 & 15.7 & NNN bend \\
\hline 945 & 20.7 & 884.4 & 13.4 & $\mathrm{C}-\mathrm{C} / \mathrm{C}-\mathrm{N}$ sym. str. \\
\hline 973.6 & 0.9 & 937.6 & 26 & $\mathrm{CH}_{2}$ rock + \\
\hline 979 & 15.7 & 1006.2 & 9 & $\begin{array}{c}\mathrm{C}-\mathrm{C} / \mathrm{C}-\mathrm{N} \text { asym. str. } \\
\mathrm{CH}_{2} \text { rock }+ \\
\mathrm{C}-\mathrm{C} / \mathrm{C}-\mathrm{N} \text { asym. str. }\end{array}$ \\
\hline 1241.5 & 0.6 & 1253.9 & 1 & $\mathrm{CH}_{2}$ twist \\
\hline 1332.6 & 145.2 & 1314.1 & 163.1 & NNN sym. str. \\
\hline 1373.7 & 10.9 & 1372 & 10.9 & $\mathrm{CH}_{2}$ wag. \\
\hline 1479.1 & 7.4 & 1476.5 & 8.4 & $\mathrm{CH}_{2}$ bend \\
\hline 2255.1 & 517.8 & 2254.3 & 450.9 & NNN asym. str. \\
\hline 2376.7 & 0.9 & 2358 & 0.6 & $\mathrm{C} \equiv \mathrm{N}$ \\
\hline 3021.4 & 15.4 & 3043.9 & 14.3 & $\mathrm{CH}_{2}$ sym. str. \\
\hline 3062.3 & 4.3 & 3135.7 & 0.4 & $\mathrm{CH}_{2}$ asym. str. \\
\hline
\end{tabular}
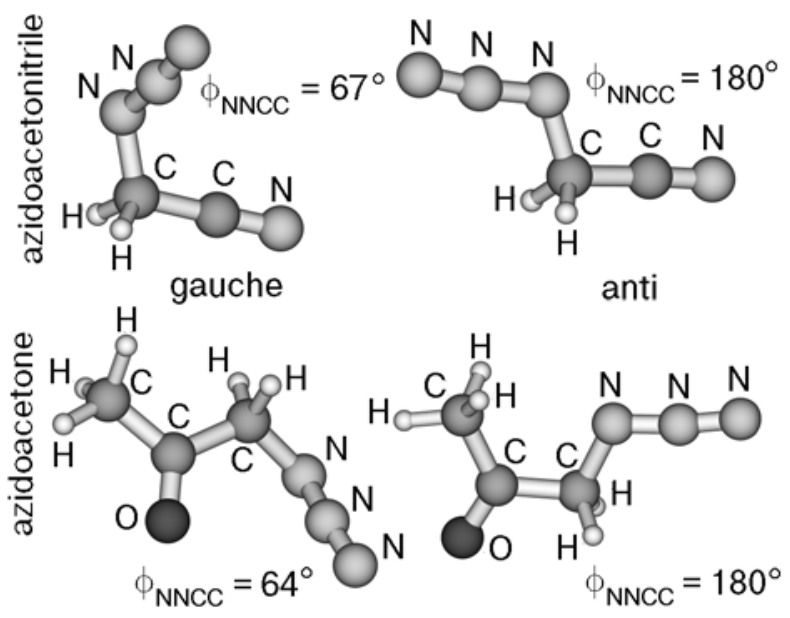

Fig. 1. Predicted structures of the gauche and anti conformers of azidoacetonitrile and azidoacetone. 
Table 2

Calculated frequencies of vibrational modes of azidoacetone conformers (method: B3LYP /6-31+G(d,p)

\begin{tabular}{|c|c|c|c|c|}
\hline \multicolumn{2}{|c|}{$\begin{array}{c}\text { Anti: dihedral angle } \\
\phi_{\mathrm{NNCC}}=180^{\circ}\end{array}$} & \multicolumn{2}{|c|}{$\begin{array}{l}\text { Gauche: dihedral } \\
\text { angle } \phi_{\mathrm{NNCC}}=64^{\circ}\end{array}$} & \multirow{2}{*}{$\begin{array}{l}\text { Approximate } \\
\text { assignment }\end{array}$} \\
\hline $\begin{array}{l}\text { frequency, } \\
\mathrm{cm}^{-1}\end{array}$ & $\begin{array}{l}\text { intensity, } \\
\mathrm{km} / \mathrm{mol}\end{array}$ & $\mid \begin{array}{c}\text { frequency, } \\
\mathrm{cm}^{-1}\end{array}$ & $\begin{array}{l}\text { intensity, } \\
\mathrm{km} / \mathrm{mol}\end{array}$ & \\
\hline 35.4 & 15.3 & 61.1 & 1.9 & \\
\hline $\begin{array}{c}46.9 \\
137.8\end{array}$ & $\begin{array}{l}1.3 \\
3.8\end{array}$ & $\begin{array}{c}81 \\
98.6\end{array}$ & $\begin{array}{l}2.5 \\
0.3\end{array}$ & $\mathrm{CH}_{3}$ torsion + ? \\
\hline 140.9 & 0 & 216.5 & 4.5 & $\mathrm{CH}_{3}$ torsion + ? \\
\hline $\begin{array}{l}262 \\
449\end{array}$ & $\begin{array}{c}10.5 \\
1.1\end{array}$ & $\begin{array}{c}274 \\
381.1\end{array}$ & $\begin{array}{l}4.9 \\
5.2\end{array}$ & \\
\hline 473.8 & $\begin{array}{l}1.1 \\
0.8\end{array}$ & 461.3 & $\begin{array}{l}3.2 \\
2.5\end{array}$ & \\
\hline $\begin{array}{l}499 \\
5561\end{array}$ & 18.4 & 556 & 6 & NNN bend + ? \\
\hline $\begin{array}{l}556.1 \\
644.2\end{array}$ & $\begin{array}{l}8.1 \\
11.7\end{array}$ & $\begin{array}{l}573.4 \\
694.3\end{array}$ & $\begin{array}{l}20.9 \\
8.2\end{array}$ & NNN bend + ? \\
\hline 799.9 & 2.3 & 794.6 & 2.2 & \\
\hline $\begin{array}{l}839.1 \\
969.6\end{array}$ & $\begin{array}{l}0.5 \\
19.5\end{array}$ & $\begin{array}{c}858.6 \\
949\end{array}$ & $\begin{array}{l}17.1 \\
23.5\end{array}$ & \\
\hline 1012.3 & 2.1 & 994.7 & 4.5 & \\
\hline $\begin{array}{l}1069.5 \\
1232.5\end{array}$ & $\begin{array}{l}0.5 \\
2.2\end{array}$ & $\begin{array}{l}1077.9 \\
1181.8\end{array}$ & $\begin{array}{l}1.6 \\
95.9\end{array}$ & $\mathrm{CH}_{2}$ twist + ? \\
\hline 1240.6 & 132.4 & 1251 & 2.9 & $\mathrm{CH}_{2}$ twist + ? \\
\hline 1342.8 & 5.8 & 1340.3 & 105.4 & $\begin{array}{l}\text { NNN sym. str. + } \\
\mathrm{CH}_{2} \text { wag. }\end{array}$ \\
\hline 1348.9 & 147.5 & 1368.1 & 32.1 & $\begin{array}{l}\text { NNN sym. str. + } \\
\mathrm{CH}_{2} \text { wag. }\end{array}$ \\
\hline 1394.6 & 45.2 & 1393.5 & 32 & $\mathrm{CH}_{3}$ umbrella mode \\
\hline 1454.5 & 8.3 & 1463.4 & 2.3 & $\begin{array}{c}\mathrm{CH}_{2} \text { bend }+\mathrm{CH}_{3} \\
\text { umbrella }\end{array}$ \\
\hline 1461.4 & 52.3 & 1469.6 & 22.5 & $\begin{array}{l}\mathrm{CH}_{2} \text { bend }+\mathrm{CH}_{3} \\
\text { umbrella }\end{array}$ \\
\hline 1468.3 & 12.8 & 1482.5 & 10.7 & $\mathrm{CH}_{3}$ str. + torsion \\
\hline 1794.3 & 207.7 & 1807.4 & 109.7 & CO str. + ? \\
\hline 2255.3 & 601.8 & 2255.9 & 522.3 & NNN asym. str. \\
\hline 3024.1 & 21.8 & 2990.8 & 31.9 & $\mathrm{CH}_{2}$ sym. str. \\
\hline 3050.2 & 0.3 & 3041.7 & 3.4 & $\mathrm{CH}_{3}$ sym. str. \\
\hline 3069.8 & 7.5 & 3104.6 & 8 & $\mathrm{CH}_{2}$ asym. str. + ? \\
\hline 3110.9 & 3.3 & 3112.3 & 7.8 & $\mathrm{CH}_{2}^{2}$ str $+\mathrm{CH}_{3}$ str. \\
\hline 3167.2 & 10 & 3161.2 & 5 & $\mathrm{CH}_{3}$ asym. str. \\
\hline
\end{tabular}

\section{Experimental results}

The infrared absorption spectra of both compounds in solid neon, argon and nitrogen were recorded in the range from 400 to $5000 \mathrm{~cm}^{-1}$, with selected regions being shown in Figs. 2 and 3. A comprehensive listing of the bands observed in our experiments, and their tentative interpretation, as well as the previous assignments by Klaeboe et al. [6] are collected in Table $3^{*}$.

Very often matrix isolation yields excellent quality spectra with sharp absorption bands, which exhibit little perturbation when compared with the gas phase. In fact, one way to judge the extent of the medium effect is to compare spectra using several different matrix materials where negligible changes from matrix to matrix usually imply also a small medium shift from gas phase to matrix, and this was one of the reasons for performing our current study.

Unfortunately, in the present case, most of the observed spectral bands are relatively broad, and they also exhibit appreciable, matrix dependent changes in terms of band width, band shape, and band intensity from matrix to matrix. Also somewhat surprisingly, the observed bands are broadest in solid neon, which usually yields the best quality spectra. One usually gets excellent quality sharp spectra when the guest fits well into the crystalline structure of the host. Thus linear compounds and cations of the type $\mathrm{X}-\left(\mathrm{C}_{2}\right)_{n}-\mathrm{Y}$, or for instance fluorinated benzene cations, yield very sharp, unperturbed spectra [12-14]. In fact, in both cases two spectrally shifted sites are observed, which were attributed to species isolated in relatively unperturbed closely packed crystalline fcc or hcp environment, respectively. The former, linear compounds replace a row in a plane of neon atoms, while the latter, benzenes, fit well into a site replacing seven atoms in a crystallographic plane. One interpretation of broad bands is an awkward fit of the relatively bulky, low symmetry guest into the solid host, preventing formation of a well-developed local crystalline structure, and resulting in poorly-defined sites, with a wide range of local geometries.

Also the theoretical computations are of less help than is often the case. Thus, as shown in Table 1, by far the strongest band computed for $\mathrm{AzN}$ is the asymmetric stretching frequency of the $\mathrm{N}_{3}$ group, predicted to lie in both conformers at about $2254 \mathrm{~cm}^{-1}$ (unscaled) with the only other fundamental frequency in this region being the almost three orders of magnitude weaker $\mathrm{CN}$ stretching frequency expected at $2358 \mathrm{~cm}^{-1}$ (2377 $\mathrm{cm}^{-1}$ in the anti compound). The experimental spectrum reveals at least seven bands, several of them quite strong, extending from 2085 to $2259 \mathrm{~cm}^{-1}$ (Fig. 2,b). Some of these bands exhibit asymmetric line shapes and additional splitting, and appreciable changes in their shapes, frequencies, and relative intensities from matrix to matrix. The corresponding $\mathrm{N}_{3}$ symmetric stretch is computed to be the second strongest absorption band. It is predicted to be in the anti and gauche conformers to be at 1333 and $1314 \mathrm{~cm}^{-1}$, respectively, with the latter value scaled by a factor of 0.96 coming very close to the observed band appearing in all three matrices between 1260-1262 $\mathrm{cm}^{-1}$ (Fig. 2,a).

* Footnotes for Table 3: i) weak bands around $2500 \mathrm{~cm}^{-1}$ and in the range of $2900-4500 \mathrm{~cm}^{-1}$ as well as matrix isolated water and $\mathrm{CO}_{2}$ bands regions have been omitted; ii) frequencies grouped in braces show fine structure of the bands; $\mathrm{m}-$ medium, s - strong, vs - very strong, the others are weak or very weak; b - broad.

${ }^{\#}$ Underlined frequencies for AzN $/ \mathrm{N}_{2}$ relate to previous assignment [6]. 
Table 3

Experimental data for azidoacetonitrile and azidoacetone isolated in $\mathrm{Ne}, \mathrm{N}_{2}$ and Ar solids*

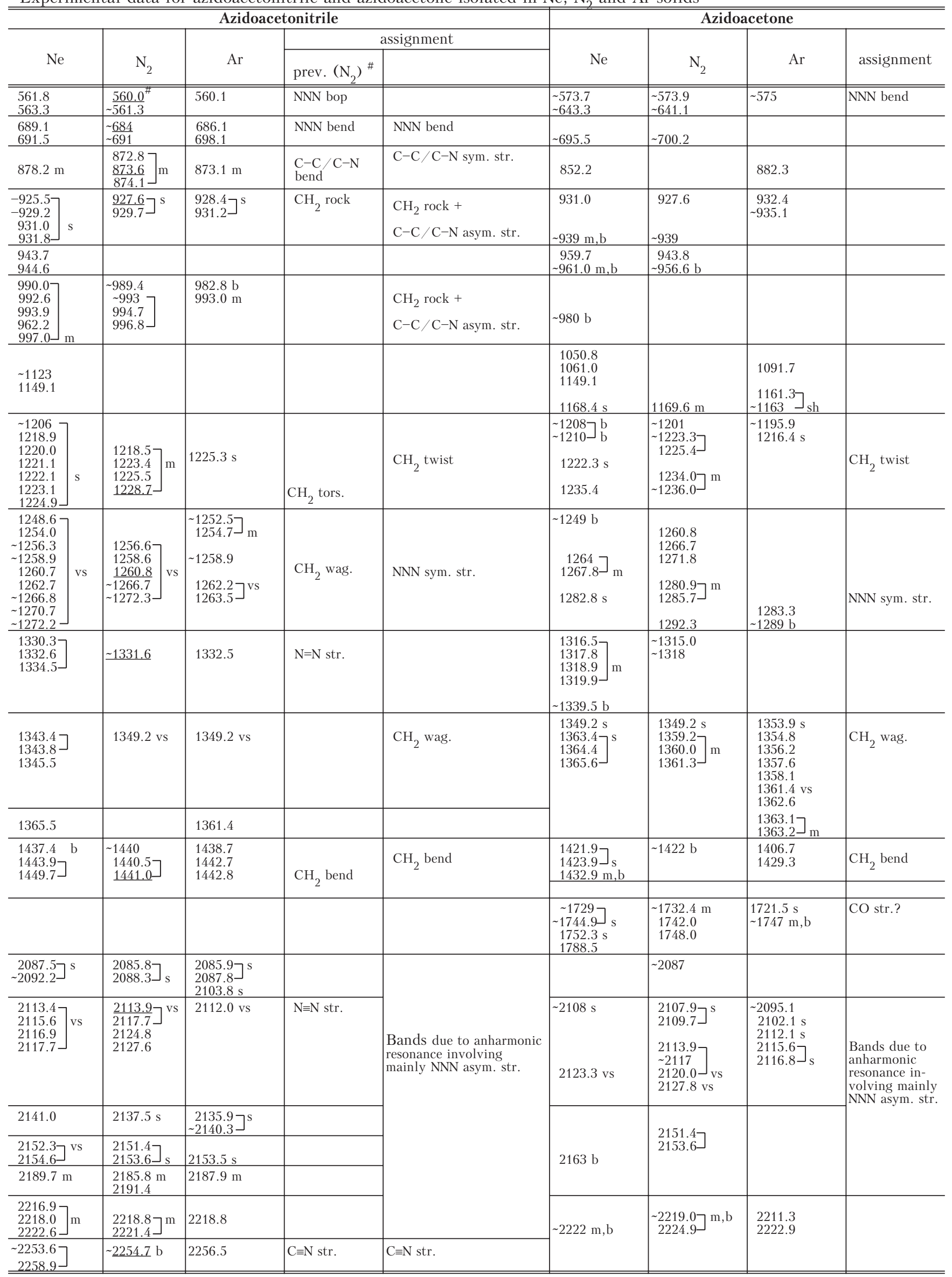



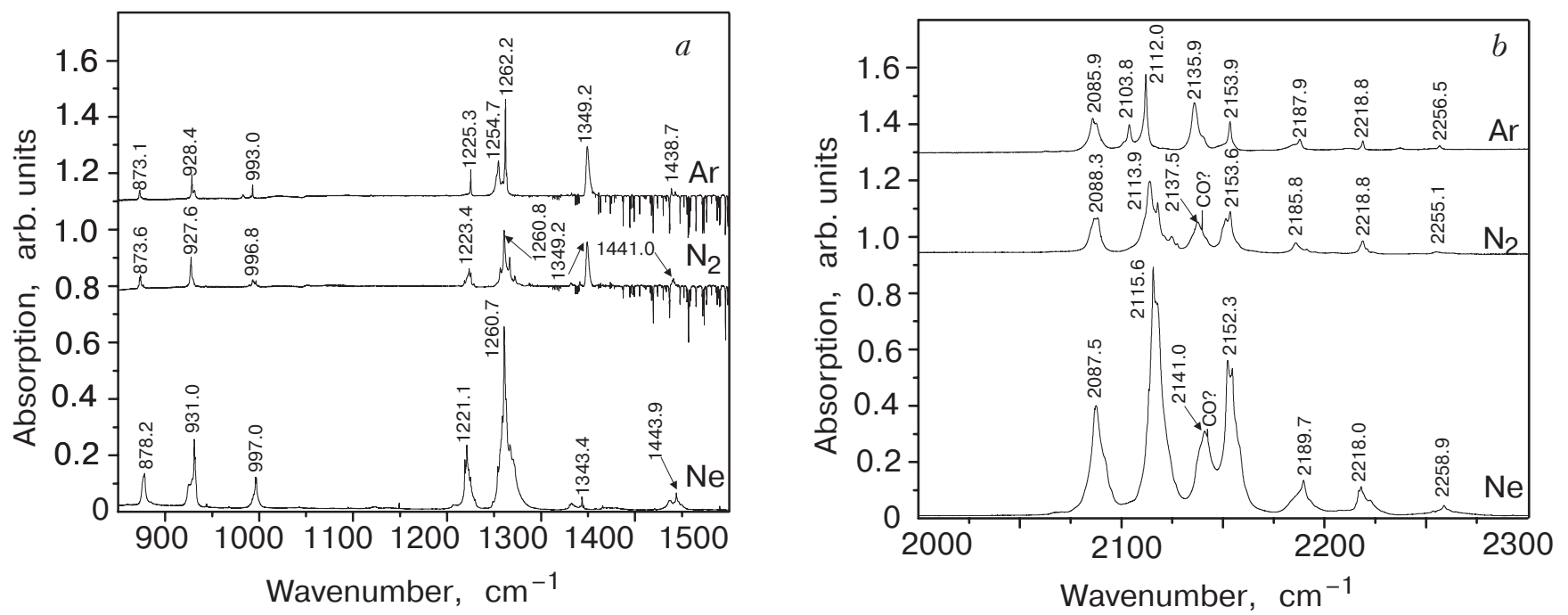

Fig. 2. Infrared spectra of azidoacetonitrile in neon, nitrogen and argon matrices («negative» lines in $\mathrm{CH}_{2}$ bend region originate from gas phase water subtraction).

The three weak to moderately intense bands appearing in all three matrices in the relatively narrow regions around 1221-1225, 1343-1349, and 1438-1444 $\mathrm{cm}^{-1}$ (Fig. 2,a) are with all probability due to the motions of the $\mathrm{CH}_{2}$ group, twist, wagging and bending, respectively, in acceptable agreement with the predicted values of 1254, 1372, and 1476 $\mathrm{cm}^{-1}$, unscaled (gauche, Table 1). These modes are computed at similar frequencies and intensities in the gauche and anti species, and therefore do not provide a useful basis for distinguishing between the two.

Most useful for discriminating between the two conformers appears to be the $700-1100 \mathrm{~cm}^{-1}$ region.
The three moderately intense bands observed in the experimental spectrum of AzN near 993-997, 928-931, and $873-878 \mathrm{~cm}^{-1}$, respectively (Fig. $2, a$ ), are in a distinctly better agreement with the comparably intense 1006, 938, and $884 \mathrm{~cm}^{-1}$ bands predicted for the gauche conformer, than with the two strong and one very much weaker anti species bands, computed to lie near 979, 974, and $945 \mathrm{~cm}^{-1}$.

The previously noted, complex structure in the region of the asymmetric $\mathrm{N}_{3}$ stretch, which is insensitive to the sample deposition rate and conditions, and which does not change upon sample annealing or photolysis is unlikely to be due to the presence of mul-
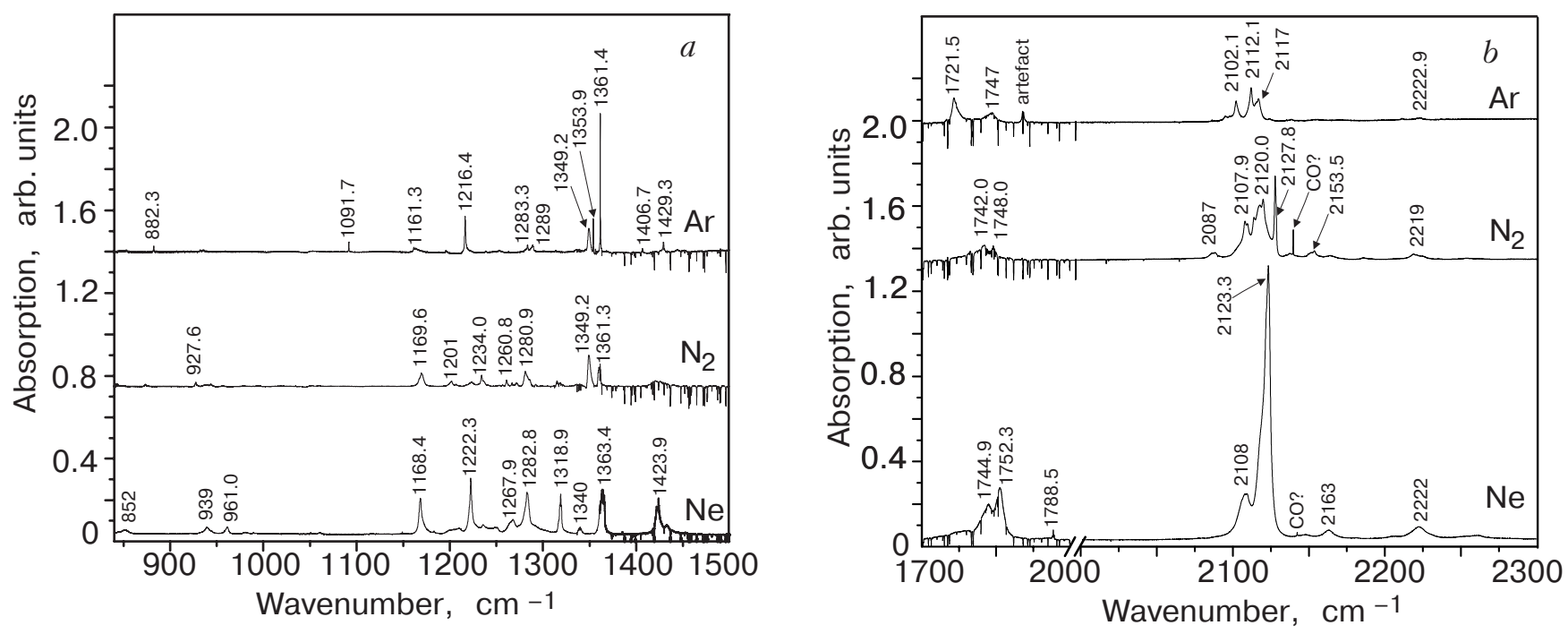

Fig. 3. Infrared spectra of azidoacetone in neon, nitrogen and argon matrices («negative» lines in CO stretch region originate from subtraction of gas phase water). No spectral features in $1800-2000 \mathrm{~cm}^{-1}$ have been observed. 
tiple conformers. The more than $150 \mathrm{~cm}^{-1}$ range of the observed bands is way too large to be attributed to matrix sites, and suggests the appearance of the spectrum is due to an intrinsic property of AzN. The molecule has a low, $C_{1}$ symmetry, and the most likely explanation of the observed multiple strong bands seems to lie in very strong Fermi resonances between the $\mathrm{N}_{3}$ asymmetric frequency, the $\mathrm{CN}$ stretching frequency, and combinations and overtones of the numerous lower frequency vibrational modes expected in this region. It should be mentioned that also a previous study [6] suggested a similar complexity in this region even in the gas phase spectrum. A rather weak band observed in all three matrices around 2255-2259 $\mathrm{cm}^{-1}$, but absent from the spectra of azidoacetone to be discussed below is undoubtedly due to the $\mathrm{CN}$ stretching vibration (Figs. 2,b and 3,b).

For the more complex azidoacetone molecule, with its twelve atoms and thirty vibrational modes, more complex infrared spectra should be expected, making their interpretation correspondingly more difficult. Similar to AzN, also in the AzC by far the most intense infrared fundamental is predicted to be the asymmetric $\mathrm{N}_{3}$ vibration, computed to lie in both conformers near $2255 \mathrm{~cm}^{-1}$ (unscaled). Also here several structured bands are found in this region, whose appearance, width and splitting changes substantially from matrix to matrix (Fig. 3,b). In neon a dominant band appears at $2123.3 \mathrm{~cm}^{-1}$ with a much weaker shoulder at $2108 \mathrm{~cm}^{-1}$ and two much weaker ones at 2163 and $2222 \mathrm{~cm}^{-1}$. All these bands are relatively symmetric, and have widths in excess of $10 \mathrm{~cm}^{-1}$. In argon and nitrogen matrices there is not one dominant band, but a more complex structure, with the strong band appearing to have split into several components with differing widths and intensities, as can be seen in Fig. 3,b.

Three other bands of the $\mathrm{AzC}$ gauche conformer are predicted to have appreciable intensities: the $\mathrm{CO}$ stretch at 1807, and bands at 1340 and $1182 \mathrm{~cm}^{-1}$, presumably the symmetric $\mathrm{N}_{3}$ stretch and a $\mathrm{CH}_{2}$ twist. These two latter modes are possibly strongly mixed, and they are computed to be somewhat shifted to 1349 and $1241 \mathrm{~cm}^{-1}$ in the higher energy anti conformer. Experimentally, in all three matrices, two bands are found in the carbonyl region, at 1752.3 and $1744.9 \mathrm{~cm}^{-1}$ in neon, 1747 and $1721.5 \mathrm{~cm}^{-1}$ in argon, and 1748 and $1742 \mathrm{~cm}^{-1}$ in nitrogen (Fig. 3,b). Besides significant changes in their frequencies from matrix to matrix, also their relative intensities are found to change, with the higher frequency band being more intense in neon, but the lowest, $1721.5 \mathrm{~cm}^{-1}$ band having the highest intensity in the argon matrix.

The assignment of the numerous lower frequency modes, which are probably in view of the low molecu- lar symmetry strongly mixed, becomes more difficult. Comparison with the AzN would seem to dictate assigning in solid neon the rather strong band at $1282.8 \mathrm{~cm}^{-1}$ to the $\mathrm{N}_{3}$ symmetric stretch, and the absorptions near 1222,1363 , and $1424 \mathrm{~cm}^{-1}$ to the twisting, wagging, and bending motions of the $\mathrm{CH}_{2}$ group (Fig. 3,a).

We have already commented above that many of the spectral lines of the matrix isolated molecules investigated here are unusually broad, and suggested that one explanation might be an awkward fit into the matrix site, preventing the formation of a well-developed crystalline structure of the host matrix in the neighborhood of the guest dopant. Another remarkable observation involves the significant shifts of individual bands from matrix to matrix, and in particular the large changes in the band widths and relative intensities. A possible interpretation of this may lie in the polar nature of the azide group. In general, matrices tend to stabilize strongly ionic or polar structures, and this stabilization will be a strong function of the polarizability of the host atoms or molecules.

Another conclusion is that in a series of annealing experiments, and photolysis of the matrices at various wavelengths, no appreciable spectral changes were detected. No new bands appeared following irradiation of the matrices, there were also no substantial changes in relative band intensities, and for a given matrix the relative intensities remained consistent from experiment to experiment. Overall, the observed spectra can be interpreted in terms of the azides being isolated in the lowest energy, gauche conformation, and no clear evidence of the simultaneous presence of several conformers was found.

\section{Summary}

The infrared spectra of organic azides, azidoacetonitrile $\left(\mathrm{N}_{3} \mathrm{CH}_{2} \mathrm{CN}\right)$ and azidoacetone $\left(\mathrm{N}_{3} \mathrm{CH}_{2} \mathrm{COCH}_{3}\right)$, isolated in low temperature solids has been measured by means of Fourier-transform technique in the range from 400 to $5000 \mathrm{~cm}^{-1}$. A comparative study in neon, argon and nitrogen matrices has shown that most of the observed spectral bands are relatively broad, exhibiting appreciable changes in terms of band width, band shape, and band intensity from host to host. Significant broadening of the recorded azides bands indicate an awkward fit of the low symmetry molecules into the solid host, resulting in a wide range of local environments. Based on the DFT calculations of IR spectra for optimized structures, the strongest absorption bands observed for both compounds were assigned to asymmetric and symmetric stretches of the $\mathrm{N}_{3}$ azide group. Strong 
Fermi resonances involving $\mathrm{N}_{3}$ asymmetric stretch, $\mathrm{CN}$ stretch and combinations and overtones of the numerous lower frequency vibrational modes can be most likely an interpretation of the strong band splittings occuring in the asymmetric stretch region of the $\mathrm{N}_{3}$ group.

\section{Acknowledgments}

Authors gratefully acknowledge the EU for financial support within the Research Training Network «Reactive Intermediates».

1. M.A Bohn, Thermochimica Acta 33, 121 (1999).

2. C. Gauthier and Y. Ramodenec, Tetrahedron 57, 7513 (2001)

3. A.E. Taubl, K. Langhans, T. Kappe, and W. Stadbauer, J. Heter. Chem. 39, 1259 (2002).

4. O.P. Korobeinichev, L.V. Kuibeida, E.N. Volkov, and A.G. Shmakov, Comb. Flame 129, 136 (2002).

5. G. Williams and C. Dsilva, Sensors and Actuators B-Chem. 30, 151 (1996).

6. P. Klaeboe, K. Kosa, C.J. Nielsen, H. Priebe, and S.H. Schei, J. Mol. Struct. 160 (3-4), 245 (1987).

7. J.M. Dyke, A.P. Groves, A. Morris, J.S. Ogden, M.I. Catarino, A.A. Dias, A.M.S. Oliveira, M.L. Costa, M.T. Barros, M.H. Cabral, and A.M. C. Moutinho, J. Chem. Phys. A103, 8239 (1999).

8. M. Algarra, A.A. Dias, M.L. Costa, P. Rodrigues, M.T. Barros, V.H.C. Lopes, M.N.D.S. Cordeiro,
G. Levita, J.M. Dyke, A. Morris, and J.S. Ogden (to be submitted)

9. M.I. Catarino, B.J. Costa Cabral, and M.L. Costa, J. Mol. Struct. (Theochem.) 397, 223 (1997).

10. K. Freudenberg, H. Eichel, and F. Leutert, Ber. Dtsh. Chem. Ges. 65, 1183 (1932).

11. Gaussian 98, Revision A.11, M.J. Frisch, G.W. Trucks, H.B. Schlegel, G.E. Scuseria, M.A. Robb, J.R. Cheeseman, V.G. Zakrzewski, J.A. Montgomery, Jr., R.E. Stratmann, J.C. Burant, S. Dapprich, J.M. Millam, A.D. Daniels, K.N. Kudin, M.C. Strain, O. Farkas, J. Tomasi, V. Barone, M. Cossi, R. Cammi, B. Mennucci, C. Pomelli, C. Adamo, S. Clifford, J. Ochterski, G.A. Petersson, P.Y. Ayala, Q. Cui, K. Morokuma, P. Salvador, J.J. Dannenberg, D.K. Malick, A.D. Rabuck, K. Raghavachari, J.B. Foresman, J. Cioslowski, J.V. Ortiz, A.G. Baboul, B.B. Stefanov, G. Liu, A. Liashenko, P. Piskorz, I. Komaromi, R. Gomperts, R.L. Martin, D.J. Fox, T. Keith, M.A. Al-Laham, C.Y. Peng, A. Nanayakkara, M. Challacombe, P.M.W. Gill, B. Johnson, W. Chen, M.W. Wong, J.L. Andres, C. Gonzalez, M. Head-Gordon, E.S. Replogle, and J.A. Pople, Gaussian, Inc., Pittsburgh PA (2001).

12. J. Agreiter, A.M. Smith, and V.E. Bondybey, Chem. Phys. Lett. 241, 317 (1995).

13. A.M. Smith, J. Agreiter, and V.E. Bondybey, Chem. Phys. Lett. 244, 379 (1995).

14. V.E. Bondybey and M. Lorenz, J. Low Temp. Phys. 122, 509 (2001) 